\title{
CLASSIFICATION OF 2-MANIFOLDS WITH SINGULAR POINTS
}

\author{
CHIEN-KE LU
}

1. Introduction. By a closed 2-manifold, or simply a 2-manifold, we mean here a two-dimensional connected finite simplicial complex every point of which has a neighborhood homeomorphic to a circular disk, that is, the interior of a circle. If there is a point not having the latter property, we call it a singular point of it.

In this paper, we shall give a complete classification (\$2) and some properties (\$3) of 2-manifolds with a single singular point. Obviously one may get one such geometrical figure by identifying certain points of a 2-manifold or several ones together. Conversely, we shall show that every such figure may be obtained in such a manner (see (2.3)).

In $\S 4$, we generalize these results to 2 -manifolds with any number of singularities.

2. The classification. The classification lies in the investigation of the nature of the neighborhoods of the singular point. Let ${ }^{1} \cdot \mathfrak{M}^{2}$ have its singular point at 0 . We first establish the following lemma.

Lemma (2.1). Any neighborhood of 0 is homeomorphic to a finite number, say $p$, of circular disks with all their centers identified. We call it a p-bundle and call 0 its center; and the boundaries of these $p$ disks are simply said to be the boundary of the p-bundle.

Proof. Consider a simplicial subdivision $\Re^{2}$ of $\cdot \mathfrak{M}^{2}$. We first note that 0 must be a vertex of $\Omega^{2}$. For, if 0 were an inner point of a 2 -simplex, then 0 could not belong to any other simplex and hence would be an ordinary point; and if it were an inner point of a 1simplex, then all the points of this 1 -simplex would be singular points for the same reason. It is also evident that 0 cannot be a vertex of a 1-simplex unless it is a vertex of a 2 -simplex.

Let 0 be a vertex of a 2 -simplex $\Re^{2}$. Then there must be many 2-simplexes including $\Re^{2}$ forming a circular disk surrounding 0 , as otherwise there would be two edges of singularities. Besides, 0 must be a vertex of another 2 -simplex, say ' $\Re^{2}$, by noting that 0 is a singular point. Hence we get another circular disk consisting of

Received by the editors September 15, 1948.

${ }^{1}$ We use the notation $\mathfrak{M}$ instead of a dot directly over $\mathfrak{M}$ for typographical convenience. 
2 -simplexes surrounding 0 . In such a way, finally, we obtain $p$ such circular disks (since $\cdot \mathfrak{M}^{2}$ is finite), and the lemma is proved.

We say $\cdot \mathfrak{M}^{2}$ has a singularity of order $p$ at $0 . \mathfrak{M}^{2}-(0)$ in general is not connected and consists of $n$ components $\mathfrak{R}_{i}^{2}(i=1, \cdots, n)$. We name $\mathfrak{R}_{i}^{2}+(0)$ a sheet of $\cdot \mathfrak{M}^{2}$. Then $\cdot \mathfrak{M}^{2}$ is the sum of these sheets with the identification of 0 . Moreover, each circular disk of 0 belongs wholly to one and only one sheet. Let us denote these sheets by $\cdot \mathfrak{M}_{i}^{2}=\mathfrak{R}_{i}^{2}+(0)(i=1, \cdots, n)$, then

$$
\begin{aligned}
\mathfrak{M}^{2} & =\sum_{i=1}^{n} \cdot \mathfrak{M}_{i}^{2}, \\
p & =\sum_{i=1}^{n} p_{i},
\end{aligned}
$$

where $p_{i}$ is the order of 0 in $\cdot \mathfrak{M}_{i}^{2}\left(p_{i}=1\right.$ in case $\cdot \mathfrak{M}_{i}^{2}$ itself is a 2 -manifold).

Hence it is sufficient for us to consider $\mathfrak{M}_{\imath}^{2}$ separately. But the structure of $\cdot \mathfrak{M}_{i}^{2}$ is quite clear; for, if we take away the $p_{i}$ circular disks surrounding 0 , the rest is a bounded 2-manifold with $p_{i}$ holes, the classification of which is already well known. ${ }^{2}$ Therefore we get:

THEOREM (2.2). Any 2-manifold with one singularity may be decomposed into the form (1), where $\mathfrak{M}_{i}^{2}$ are sheets, that is, bounded 2-manifolds with $p_{i}$ holes adjoined with a $p_{i}$-bundle having its boundary identified with the boundaries of these holes; and all the centers of these bundles are to be identified.

We may, however, consider the $p$-bundle separately as $p$ circular disks and identify each of their circumferences with each of the boundaries of the holes. Thus we get 2-manifolds, and then identify the $p$ centers. Hence we obtain:

THEOREM (2.3). Every 2-manifold with one singular point is the sum of a finite number of 2-manifolds each with some points identified all together.

The preceding two theorems lead us to obtain a 2-manifold with a single singular point from bounded and closed 2-manifolds respectively. In practice the latter is much more useful than the former.

3. Simple properties. The most evident property of 2-manifolds with one singularity is contained in the following theorem.

TheOREM (3.1). $\cdot \mathfrak{M}^{2}$ and $\cdot \mathfrak{M}^{* 2}$ are homeomorphic if and only if they

${ }^{2}$ Cf., for example, Seifert-Threlfall, Lehrbuch der Topologie, 1934, §40. 
have the same structures, hence necessarily, after suitably arranging their sheets,

$$
\left(p_{i}\right)=\left(p_{i}^{*}\right)
$$

(consequently $n=n^{*}, p=p^{*}$ ).

But we should notice that (3) is not a sufficient condition for $\cdot \mathfrak{M}^{2}$ and $\mathfrak{M}^{* 2}$ to be homeomorphic, since the corresponding 2-manifolds constituting them may not be homeomorphic. We also note that the property of orientability is preserved:

THEOREM (3.2). A 2-manifold with one singularity is orientable if and only if all the 2-manifolds constituting it are orientable.

Now we come to prove the important theorem:

THEOREM (3.3). The (integral) homology group $\mathfrak{h}$ of any dimension of a 2-manifold with one singularity is the direct sum of those of its sheets. The homology group of a sheet $\cdot \mathfrak{M}_{i}^{2}$ is the same as that of the 2-manifold $\mathfrak{M}_{i}^{2}$ except for dimension 1 , where $\mathfrak{M}_{i}^{2}$ is the 2-manifold which is the same as $\cdot \mathfrak{M}_{i}^{2}$ but without those $p_{i}$ points to be identified in $\cdot \mathfrak{M}^{2}$ being identified in $\mathfrak{M}_{\mathfrak{i}}^{2}$. For dimension 1 , if $\cdot \mathfrak{M}_{\mathfrak{i}}^{2}$ and $\mathfrak{M}_{\mathfrak{i}}^{2}$ have the homology groups $\cdot \mathfrak{h}_{\mathfrak{r}}^{1}$ and $\mathfrak{h}_{\mathbb{1}}^{1}$ respectively, then

$$
\cdot \mathfrak{h}_{i}^{1}=\mathfrak{h}_{i}^{1}+\left(p_{i}-1\right) \mathfrak{g}
$$

where $\mathrm{kg}$ represents the direct sum of $k$ free cyclic groups $\mathfrak{g}$.

Proof. Let us consider the last statement only as the others are obvious. In case $p_{i}=1,(4)$ is trivial.

Suppose $0_{1}, \cdots, 0_{p_{i}}$ are the points of $\mathfrak{M}_{i}^{2}$ which are to be identified in $\mathfrak{M}_{\mathfrak{i}}^{2}$. Take a sufficiently small simplicial subdivision of $\mathfrak{M}_{\mathfrak{i}}^{2}$ such that all these points are vertices, and it induces a simplicial subdivision on $\cdot \mathfrak{M}_{i}^{2}$. Then any 1 -cycle $Z^{1}$ on $\mathfrak{M}_{i}^{2}$ is either a 1 -cycle on $\mathfrak{M}_{i}^{2}$ or a broken line joining two $0^{\prime}$ 's, say $0_{j}$ and $0_{k}$. In the latter case $Z^{1}$ is neither homologous nor division homologous to zero. For if $m Z^{1} \sim 0$ $(m \neq 0)$, then there would exist a 2 -chain $C^{2}$ whose boundary $\partial C^{2}=m Z^{1}$ and thence

$$
\partial \partial C^{2}=m \partial Z^{1}=m\left( \pm 0_{j} \pm 0_{k}\right) \neq 0,
$$

which is a contradiction. Hence $Z^{1}$ as an element of $\cdot \mathfrak{h}_{i}^{1}$ generates a free cyclic group.

We then join $0_{1}$ to the other 0 's and get $p_{i}-1$ broken lines, each of which generates a free cyclic group since no two of them are homologous or division homologous to zero by the same reason. 
Any broken line between $0_{j}$ and $0_{k}$ may be replaced by an algebraic sum of two broken lines starting from $0_{1}$ and ending in $0_{j}, 0_{k}$ respectively, and a 1-cycle through these three points on $\mathfrak{M}_{\mathfrak{i}}^{2}$ will be discussed below.

A 1-cycle on $\mathfrak{M}_{i}^{2}$ not passing through any 0 is not influenced in constructing $\cdot \mathfrak{M}_{i}^{2}$, while one passing any 0 , say $0_{k}$, may be modified by omitting the two edges through it and adding the third edge of the 2 -simplex that is incident with $0_{k}$ as well as the two edges through $0_{k}$ (see (2.1)). Hence the homology classes made by the 1-cycles on $\mathfrak{M}_{\mathfrak{i}}^{2}$ are unchanged on $\mathfrak{M}_{\mathfrak{i}}^{2}$. Thus (3.3) is established.

Corollary (3.4).

$$
\cdot \mathfrak{h}^{1}=\sum_{i=1}^{n} \cdot \mathfrak{h}_{i}^{1}+(p-n) \mathfrak{g} .
$$

This shows us a method for constructing a 2-complex with any preassigned Betti number whatever.

Analogously, we have the following theorem.

THEOREM (3.5). The fundamental group of a 2-manifold with one singularity is the free product of those of its sheets, and the fundamental group $\mathfrak{f}_{i}$ of a sheet $\cdot \mathfrak{M}_{i}^{2}$ is the free product of the fundamental group $\mathfrak{f}_{i}$ of $\mathfrak{M}_{i}^{2}$ and $p_{i}-1$ free cyclic groups. ${ }^{3}$

4. Generalizations. By the finiteness of a 2-manifold it is evident that the number of singular points on it, if any, is finite. Let $' 0,{ }^{\prime \prime} 0, \cdots,{ }^{(m)} 0$ be the only singular points on a 2 -manifold $\cdot \mathfrak{M}^{2}$.

Lemma $(2.1)$ is valid for each ${ }^{(j)} 0(j=1, \cdots, m)$ and we may speak of the order at ${ }^{(j)} 0$, say ${ }^{(j)} p$. The generalized Theorems $(2.2)$ and (2.3) have their natural forms, the latter of which we state as follows.

THEOREM (4.1). $\cdot \mathfrak{M}^{2}$ is the sum of a finite number of 2-manifolds $\cdot \mathfrak{M}_{i}^{2}(i=1, \cdots, n)$ on which ${ }^{(j)} p_{i}$ points are identified to the point (i) $0(j=1, \cdots, m)$.

Moreover,

$$
p_{i}=\sum_{j=1}^{m}{ }^{(j)} p_{i}, \quad{ }^{(j)} p=\sum_{i=1}^{n}{ }^{(j)} p_{i}, \quad p=\sum_{i=1}^{n} p_{i}=\sum_{j=1}^{m}{ }^{(j)} p,
$$

where $p_{i}$ and $p$ are defined as the orders of $\cdot \mathfrak{M}_{i}^{2}$ and $\cdot \mathfrak{M}^{2}$ respectively.

3 We may first prove (3.5) and so (3.3) follows immediately by a relation between the homology group and the fundamental group, cf. ibid. p. 173. 
Theorem (3.1) now takes the form:

THEOREM (4.2). $\cdot \mathfrak{M}^{2}$ and $\cdot \mathfrak{M}^{* 2}$ are homeomorphic if and only if they have the same structures, hence necessarily, after suitably arranging their sheets and the order of their singular points,

$$
\left.{ }^{\left({ }^{(j)}\right.} p_{i}\right)=\left({ }^{(j)} p_{i}^{*}\right)
$$

(consequently $m=m^{*}, n=n^{*}, p=p^{*}$ ).

Theorem (3.2) is true in its original form. We establish now the following theorem.

Theorem (4.3). The homology group $\cdot \mathfrak{h}_{i}^{1}$ of $\cdot \mathfrak{M}^{2}$ is given by

$$
\cdot \mathfrak{h}_{i}^{1}=\mathfrak{h}_{\mathfrak{i}}^{1}+\left(p_{i}-m\right) \mathfrak{g},
$$

where $\mathfrak{h}_{i}^{1}$ is the homology group of $\mathfrak{M}_{i}^{2}$, the 2-manifold which is the same as $\cdot \mathfrak{M}_{i}^{2}$ but without any points being identified.

Proof. For each $j$ we consider the ${ }^{(j)} p_{i}$ points to be identified to (i) 0 as in the proof of (3.3), that is, join broken lines from one of them to all the others. They are 1-cycles on $\mathfrak{M}_{4}^{2}$, each of which generates a free cyclic group in $\mathfrak{h}_{\mathfrak{i}}^{1}$ and any two of which are neither homologous nor division homologous to zero on $\cdot \mathfrak{M}^{2}$. Any 1-cycle on $\cdot \mathfrak{M}_{i}^{2}$ may be replaced by an algebraic sum of these broken lines and a 1-cycle on $\mathfrak{M}_{i}^{2}$. Hence by the same reason as in (3.3), from the first equation of (5) we have (7), and thus the theorem is proved.

In order to get the homology group $\mathfrak{h}^{1}$ of $\cdot \mathfrak{M}^{2}$, we again introduce a lemma which may be readily proved.

Lemma (4.4). If $\Omega$ is a connected simplicial complex of any dimension and $P_{1}, \cdots, P_{k}$ are $k$ arbitrary distinct points on $i$, and $\Omega^{*}$ is the complex made by $\Omega$ in addition with the 1 -simplexes $\left(P_{1} P_{2}\right),\left(P_{1} P_{3}\right), \cdots$ $\left(P_{1} P_{k}\right)$ (not in $\left.\Re\right)$; then

$$
\mathfrak{h}^{* \mathfrak{1}}=\mathfrak{h}^{1}+(k-1) \mathfrak{g},
$$

or

$$
\mathfrak{h}^{\mathfrak{1}}=\mathfrak{h}^{* 1}-(k-1) \mathfrak{g},
$$

where $\mathfrak{h}^{1}$ and $\mathfrak{h}^{* 1}$ are homology groups of $\Omega$ and $\Omega^{*}$ respectively and the minus sign indicates a difference group.

Eventually, we have the following theorem.

Theorem (4.5). The homology group $\cdot \mathfrak{h}^{1}$ of $\cdot \mathfrak{M}^{2}$ may be written as 


$$
\cdot \mathfrak{h}^{1}=\sum_{i=1}^{n} \mathfrak{h}_{i}^{1}+(p-n-m+1) \mathfrak{g} .
$$

Proof. In constructing 1-simplexes $\left({ }^{\prime} 0^{\prime \prime} 0\right), \cdots,\left({ }^{\prime} 0^{(m)} 0\right)$ (not belonging to $\cdot \mathfrak{M}^{2}$, we get $\cdot \mathfrak{M}^{* 2}, \mathfrak{M}_{1}^{* 2}, \cdots$ as in the lemma. By $(7)$ and (8), we have

$$
\cdot \mathfrak{h}_{i}^{* 1}=\mathfrak{h}_{i}^{1}+\left(p_{i}-1\right) \mathfrak{g}
$$

where $\cdot \mathfrak{h}_{i}^{* 1}$ is the homology group of $\cdot \mathfrak{M}_{t}^{* 2}$. The newly constructed simplexes form a connected 1-complex whose 1-dimensional homology group contains the identity only. Therefore from a famous theorem (cf. Seifert-Threfall, p. 179), by (5) we get

$$
\cdot \mathfrak{h}^{* 1}=\sum_{i=1}^{n} \mathfrak{h}_{i}^{1}+(p-n) \mathfrak{g},
$$

where $\mathfrak{h}^{* 1}$ is the homology group of $\cdot \mathfrak{M}^{* 2}$. Therefore (9) is finally established in virtue of (11) and (8)'.

Theorem (3.5) may be extended analogously.

NATIONAL WUHAN UnIVERSity

\section{A NOTE ON EQUICONTINUITY}

M. K. FORT, JR.

During a recent seminar discussion of his paper Transitivity and equicontinuity [1], ${ }^{1}$ W. H. Gottschalk proposed the following question:

"Is the center of every algebraically transitive group of homeomorphisms on a compact metric space equicontinuous?"

An affirmative answer to the above question is given in this note.

1. Definitions. We let $X$ and $Y$ be compact metric spaces and let $d$ be the metric for $Y$.

A set $F$ of functions on $X$ into $X$ is algebraically transitive if corresponding to each pair $p$ and $q$ of points in $X$ there exists $f \in F$ such that $f(p)=q$.

A sequence $\left[g_{n}\right]$ of functions on $X$ into $Y$ converges to a function

Presented to the Society, November 27, 1948; received by the editors August 10, 1948.

${ }^{1}$ Numbers in brackets refer to the bibliography at the end of the paper. 